\title{
Los agrupamientos escolares: expectativas, prácticas y experiencias
}

\section{Students grouping: expectations, practices and experiences}

\author{
Alba Castejón Company \\ alba.castejon@uab.cat \\ Universidad Autónoma de Barcelona. España \\ Jordi Pàmies Rovira (iD) \\ jordi.pamies@uab.cat \\ Universidad Autónoma de Barcelona. España
}

\section{Resumen}

El agrupamiento por niveles en el interior de los centros es una estrategia que se practica en numerosos sistemas educativos, a pesar de que se estructuren bajo los principios de la comprensividad. Este es el caso de Cataluña donde, según PISA 2015, un 26\% del alumnado está escolarizado en centros de secundaria que agrupan a sus estudiantes por niveles en todas las asignaturas (un 6\% en el conjunto de España). Este artículo tiene como objetivo explorar las prácticas de agrupamiento a partir de los datos obtenidos en una etnografía llevada a cabo en un instituto de educación secundaria en Cataluña situado en un contexto de alta complejidad social. La investigación ofrece resultados concluyentes respecto a las formas en que las prácticas de agrupamiento, sujetas a las creencias y expectativas docentes, condicionan las experiencias escolares de los y las jóvenes y contribuyen a naturalizar las situaciones de desventaja educativa y social del alumnado más desfavorecido.

Palabras clave: Agrupamientos escolares; creencias y expectativas docentes; educación secundaria obligatoria; segregación escolar; desventaja educativa.

\section{Abstract}

Grouping students by ability inside schools is a strategy that is spread in many educational systems, although they are under the principles of comprehensiveness. This is the case of Catalonia where, according to PISA $2015,26 \%$ of students are enrolled in secondary schools that group their students by ability in all subjects $(6 \%$ on average in Spain). This article aims to explore the grouping practices from the data obtained in an ethnography carried out at a secondary school in Catalonia located in a highly disadvantaged context. The research offers conclusive results regarding the ways in which grouping practices, subject to teachers' beliefs and expectations, condition young people's experiences, and contribute to naturalize situations of educational and social disadvantage of the most disadvantaged students.

Keywords: Grouping practices; teachers' beliefs and expectations; compulsory secondary education; school segregation; educational disadvantage.

Cómo referenciar este artículo / How to reference this article:

Castejón, A., \& Pàmies, J. (2018). Los agrupamientos escolares: expectativas, prácticas y experiencias. Tendencias Pedagógicas, 32, 49-64. doi: 10.15366/tp2018.32.004 


\section{Introducción}

A lo largo del siglo XX la mayoría de países europeos sufrieron importantes cambios educativos, marcados esencialmente por una expansión que buscaba la escolarización universal. La mayor parte de las reformas escolares estuvieron guiadas por el principio de comprensividad, idea que promueve una escolarización obligatoria para todos "bajo un mismo techo", esto es, una escuela integrada e indiferenciada tanto para la educación primaria como la secundaria inferior (Husén, 1973).

Aunque la implantación del sistema comprensivo ha sido desigual en los diversos contextos nacionales, los estudios aportan resultados concluyentes acerca de las oportunidades que ofrecen las diversas modalidades. Así, en los últimos años, uno de los temas que más relevancia ha adquirido a nivel internacional ha sido el debate sobre las formas de diferenciación del alumnado a lo largo de su trayectoria educativa en itinerarios educativos diversificados, y su impacto en la reproducción de las desigualdades. Ahora bien, no ha sido menor el debate acerca de las formas que han adquirido los procesos de selección dentro de los propios centros a través de la estratificación escolar interna. Como ya mostró el trabajo inicial de Oakes (1982) compartir la misma escuela puede significar "compartir el mismo techo" pero no las mismas experiencias, una consideración que supone abordar las formas de agrupamiento del alumnado en la propia institución escolar.

Estudiar el agrupamiento del alumnado en la educación secundaria obligatoria es, especialmente en el contexto catalán, un aspecto relevante. En primer lugar, porque a pesar de que el sistema educativo se estructure según los principios de la comprensividad, la autonomía escolar otorga libertad a los centros educativos para que puedan agrupar al alumnado según las necesidades que consideren (Castejón, 2017). En segundo lugar, porque la ambigüedad de la normativa que regula las formas de agrupamiento se traduce en la aplicación de prácticas muy diversas. Y en tercer lugar, porque el número de investigaciones en este sentido en el contexto catalán y español resultan más bien escasas (Carrasco et al., 2011; Castejón, 2017; González-Motos, 2012; Pàmies, 2013; Ponferrada, 2009; Rubio, 2013; Serra, 2001). De hecho, si bien no se disponen de datos oficiales que permitan conocer las principales medidas que adoptan los institutos en esta materia, según datos de la última edición de PISA (PISA 2015), en Cataluña un 26\% del alumnado está escolarizado en centros que agrupan a sus estudiantes por niveles en todas las asignaturas (un $6 \%$ en el conjunto de España), mientras que un $44 \%$ lo hace en centros donde se agrupa por niveles en algunas materias (un 34\% en el conjunto de España). Estos porcentajes sitúan a Cataluña como el territorio español donde más se utiliza -con diferencia- el agrupamiento por niveles.

Este artículo tiene como objetivo explorar y analizar las prácticas de agrupamiento del alumnado y su relación con las creencias y expectativas docentes. Busca, asimismo, profundizar en la comprensión de las dinámicas escolares y entender las formas en que estas prácticas condicionan las experiencias de las y los jóvenes. Los datos analizados proceden de un estudio etnográfico, en el marco de una tesis doctoral, realizada durante el curso 2014-15 en un instituto de educación secundaria en un contexto de alta complejidad social de la Región Metropolitana de Barcelona.

\section{Clasificando al alumnado: el agrupamiento por niveles}

La delimitación conceptual de las prácticas relativas al agrupamiento del alumnado resulta, a menudo, un tanto confusa. Esto es debido, básicamente, a la gran diversidad de formas que esta medida organizativa puede tomar en los diferentes sistemas educativos o incluso entre centros, y por las diferentes nomenclaturas dadas a prácticas de carácter similar. No obstante, se pueden distinguir aquellas prácticas o estrategias que se basan en una diferenciación del alumnado, y aquellas que, al contrario, pretenden proporcionar una educación común a todos los jóvenes hasta etapas avanzadas del sistema educativo. En el segundo caso, todos los centros educativos siguen un mismo currículum y el agrupamiento del alumnado no se realiza siguiendo criterios de homogeneidad académica, sino al contrario, los grupos son construidos de forma heterogénea incluyendo estudiantes con supuestas capacidades o rendimientos diversos.

Entre los sistemas que utilizan formas de diferenciación del alumnado se pueden distinguir 
aquellos en que la estratificación es una característica sistémica (es decir, de la propia estructura del sistema educativo) o los sistemas en que la diferenciación y las decisiones asociadas a ésta se producen a nivel de centro educativo. El primer tipo de diferenciación se da en aquellos sistemas en que existen itinerarios diferenciados desde edades tempranas (Alemania, Austria, Luxemburgo, Países Bajos, Bélgica, Hungría o Suiza), reconocidos como tales en la estructura del sistema educativo, y en que el currículo y los objetivos son diferentes entre itinerarios, que suelen dividirse en académico, técnico y/o vocacional (Dupriez, 2010).

Por otro lado, el agrupamiento homogéneo del alumnado, también llamado agrupamiento por niveles o por capacidades, es la práctica de clasificar a los alumnos, en el interior de los centros educativos, en diferentes grupos de enseñanza-aprendizaje según criterios vinculados a su rendimiento o sus supuestas capacidades (Dupriez, 2010; Gamoran, 1992; Slavin, 1988). Los tipos de agrupamiento pueden variar sustantivamente dependiendo de los objetivos que se persigan, de su rigidez, de su estabilidad, o de sus consecuencias en términos de acreditación. Si bien no existe una taxonomía estandarizada sobre las formas de organización del alumnado en los grupos escolares, las diferencias en el tipo de agrupamiento homogéneo se dan fundamentalmente en base a su alcance y estabilidad (Kutnick et al., 2005; Sukhnandan y Lee, 1998). Esto es, en algunos casos los alumnos son asignados en un grupo-clase en el que permanecen en todas o la mayoría de las asignaturas, de forma que una única decisión determina la posición del estudiante para la gran mayoría de horas lectivas; en otros casos, los alumnos se agrupan de forma distinta para cada una de las asignaturas, de manera que un alumno puede estar asignado en un nivel alto o bajo dependiendo de la asignatura, y sus compañeros pueden variar en cada uno de los grupos.

En cualquier caso, aunque bajo la denominación de agrupamiento por niveles (o en inglés, ability grouping o tracking) se incluyen prácticas que difieren entre sí, todas ellas comparten el hecho de que el alumnado es clasificado, para su instrucción, según su rendimiento o sus capacidades; es decir, se trata del método que consiste en enseñar en un mismo espacio a estudiantes que se considera que "funcionan" de manera similar en los logros de aprendizaje (Abadzi, 1985).

La diferenciación académica en general, y el agrupamiento homogéneo o por niveles en particular, se ha estudiado de forma extensa en la literatura internacional, que ha puesto de manifiesto que ésta es una práctica que está ampliamente arraigada en algunos sistemas, y que sus efectos - académicos, sociales, emocionales- resultan en especial negativos para el alumnado situado en posiciones económicas desfavorecidas y en niveles bajos de rendimiento (Dupriez, 2010; Dupriez y Dumay, 2006; Gamoran, 2009; Horn, 2009). De hecho, esta estrategia se ha asociado en numerosas ocasiones con las desigualdades educativas, no solamente con su reproducción, sino como mecanismo para su amplificación. Por estas razones, interrogarse sobre cómo funcionan los agrupamientos del alumnado, por qué se aplican, qué consecuencias tienen o qué mecanismos se activan a la hora de explicar sus impactos, es fundamental para comprender de qué manera se configuran las desigualdades educativas. Las secciones siguientes muestran resultados empíricos en esta línea.

\section{Metodología}

Los datos que se presentan en este artículo proceden de una etnografía escolar llevada a cabo en un instituto de educación secundaria de clase trabajadora de la Región Metropolitana de Barcelona.

La investigación ha abordado tres objetivos específicos, a saber: 1) estudiar la relación entre las prácticas de agrupamiento homogéneo y las expectativas y creencias docentes; 2) analizar las prácticas docentes de aula en cada uno de los grupos de nivel y 3) comprender la experiencia educativa de los y las jóvenes en estos grupos.

El trabajo etnográfico ha permitido aprehender los significados y las redes de sentido subyacentes a la práctica escolar (Díaz de Rada, 2006; Geertz, 1990; Pàmies, Carrasco y Casalta, 2014; Wolcott, 1993) y comprender, desde la interpretación contextualizada de las prácticas e interacciones, de qué manera se construyen y se naturalizan los procesos educativos, se reparten las oportunidades y se 
construyen las exclusiones y las desigualdades escolares.

El trabajo de campo se inició en octubre de 2014 y se prolongó hasta finales de julio de 2015, desarrollándose tres días por semana, principalmente durante el horario lectivo. Si bien la principal técnica de recogida de información fue la observación participante, también se llevaron a cabo entrevistas semiestructuradas, cuestionarios, y análisis documental. Durante este periodo se elaboró un diario de campo, en el que se anotaron las observaciones realizadas, las interacciones entre alumnado, entre docentes, y entre alumnado y docentes; en el que se reprodujeron comentarios y conversaciones informales con los diversos actores educativos; y donde se anotaron sugerencias y comentarios que permitieron perfilar la construcción de los instrumentos de recogida sistemática de información utilizados.

Aunque el centro educativo en su conjunto fue el campo de observación, se estimó necesario establecer escenarios acotados al interior del mismo. Así, la investigadora se incorporó al funcionamiento del centro a partir de la asistencia regular a las clases y actividades de $3^{\circ}$ de la ESO, para promover el contacto regular y prolongado con un grupo concreto de docentes y alumnos, con un doble objetivo. El primero, poder aprehender las dinámicas en el aula de tal forma que fuese posible distinguir las situaciones "excepcionales" de aquellas "habituales" representativas de las interacciones cotidianas, adquiriendo una comprensión de las relaciones y procesos de socialización escolar (Lahire, 2008). El segundo, romper la barrera como "intrusa" para que los diferentes agentes educativos se fuesen "olvidando poco a poco" de su presencia.

Durante el último mes de estancia en el instituto, se llevaron a cabo entrevistas en profundidad con el alumnado (21), el profesorado de $3^{\circ}$ de la ESO (7) y otros profesionales del centro y de la administración educativa (6), así como se aplicaron dos cuestionarios, uno destinado a todos los docentes de la ESO (29 respuestas, 55\% tasa de respuesta), y otro para el alumnado de $3^{\circ}$ y $4^{\circ}$ de la ESO (137 respuestas, $88 \%$ tasa de respuesta). Todos los instrumentos fueros creados ad hoc para esta investigación. La información recogida a partir de la observación participante, de las entrevistas semiestructuradas y del análisis instrumental se analizaron mediante el software de análisis cualitativo Atlas ti. A partir de los códigos establecidos en base al modelo de análisis se codificaron los datos. Los cuestionarios del alumnado fueron tratados con el software de análisis cuantitativo SPSS. El uso de diversidad de técnicas permitió la triangulación metodológica de la información obtenida, a partir de la codificación y análisis, y su posterior comparación (Flick, 2004).

Al inicio de la investigación se obtuvo consentimiento institucional para el desarrollo de la misma, y se administró una hoja de autorización familiar a todo el alumnado que participó en el cuestionario y en las entrevistas, informando de su participación en la investigación y requiriendo la autorización materna, paterna o de los tutores legales para que, en el caso de las entrevistas, pudiesen ser registradas. Asimismo, la confidencialidad y el anonimato, tal y como se aseguró a los actores educativos, están garantizados. Por ello mismo, todos los nombres -tanto de personas como de lugares- han sido modificados y se ha omitido cualquier información que pueda representar un riesgo para la identificación del instituto o de los participantes en la investigación. Todos los nombres que aparecen en el artículo son, pues, pseudónimos.

\subsection{Presentación del Instituto del Canal}

El Instituto del Canal se encuentra en una gran ciudad (más de 200.000 habitantes a 1 de enero de 2016, según datos del Ayuntamiento) de la Región Metropolitana de Barcelona. Está situado en un barrio de la ciudad que forma parte de los distritos periféricos que crecieron de manera exponencial con la inmigración española de la segunda mitad del siglo XX y que en la actualidad han sido destino de la inmigración internacional. 
El Instituto del Canal se creó el 1982 como Instituto de Formación Profesional y en la actualidad imparte los niveles de Educación Secundaria Obligatoria (ESO), Bachillerato, Ciclos Formativos (CF) de Grado Medio y Superior, y Programas de Formación e Inserción (PFI). En el curso 20132014 en el centro se escolarizaban casi un millar de estudiantes, de los cuales 374 eran de ESO. El centro está considerado, por parte de la administración educativa de tipología $\mathrm{C}$, es decir, de máxima complejidad social.

Más allá de los indicadores que proporciona la inspección educativa, y como ya se ha explicado en la metodología, se estimó oportuno recoger, mediante un cuestionario al alumnado de $3^{\circ}$ y $4^{\circ}$ de la ESO, un conjunto de datos que permitiesen caracterizar de manera más precisa la composición social del centro. Algunos de estos indicadores se recogen en la Tabla 1. En relación con el nivel de estudios de los progenitores, buena parte del alumnado procede de familias con un capital instructivo bajo: las familias que no han alcanzado más de la educación secundaria son casi el 43\%, de las cuales casi un $20 \%$ tienen sólo estudios primarios. Asimismo, en el instituto hay una presencia importante de alumnado inmigrante, mayoritariamente de primera generación, es decir, que tanto ellos como sus padres han nacido fuera del territorio español. Por lo referente a la región de origen de este alumnado, la mayoría procede de países latinoamericanos (principalmente de Bolivia y Ecuador) mientras que más de un tercio procede de Marruecos.

Tabla 1

Nivel de estudios más alto de los progenitores, estatus migrante del alumnado y región de procedencia del alumnado inmigrante. Porcentaje de alumnos

\begin{tabular}{|c|c|c|}
\hline & & Instituto del Canal \\
\hline \multirow{5}{*}{ Nivel de estudios progenitores } & Educación primaria o menos & $19 \%$ \\
\hline & Educación secundaria & $24 \%$ \\
\hline & Ciclos Formativos o Bachillerato & $28 \%$ \\
\hline & Universidad & $15 \%$ \\
\hline & No sabe ${ }^{1}$ & $14 \%$ \\
\hline \multirow{3}{*}{ Estatus migrante } & Autóctonos & $64 \%$ \\
\hline & Inmigrantes de primera generación & $34 \%$ \\
\hline & Inmigrantes de segunda generación & $2 \%$ \\
\hline \multirow{4}{*}{ Región de procedencia alumnado inmigrante } & Latinoamérica & $54 \%$ \\
\hline & Marruecos & $34 \%$ \\
\hline & África Subsahariana & $2 \%$ \\
\hline & Otros & $10 \%$ \\
\hline
\end{tabular}

Fuente: elaboración propia a partir del cuestionario de estudiantes de $3^{\circ}$ y $4^{\circ}$ de la ESO del Instituto del Canal.

\section{Resultados}

\subsection{Los argumentos legitimadores del agrupamiento por niveles}

En el instituto del Canal existen una serie de argumentos explícitos, compartidos por la gran mayoría del profesorado, que "defienden" la legitimidad del agrupamiento por niveles. Estos argumentos, se articulan en los discursos del equipo directivo y del profesorado, así como en el Proyecto Educativo de Centro, aunque como ha mostrado el trabajo de campo, más allá de estas argumentaciones emergen lógicas subyacentes, implícitas, que explican las formas y los significados de aplicación de esta práctica organizativa.

a) Un objetivo explicito: atender a todo el alumnado

\footnotetext{
${ }^{1}$ En la pregunta acerca del nivel de estudios de los progenitores, se introdujo una posibilidad de respuesta que era «no estoy seguro/a, no lo sés. La finalidad de introducir esta opción era diferenciar las respuestas en blanco, que se han tratado como valores perdidos, del desconocimiento de la información.
}

Tendencias Pedagógicas, 32, 2018, pp. 49-64. DOI: 10.15366/tp2018.32.004 
Un primer argumento explícito que se utiliza para justificar el agrupamiento por niveles es la voluntad de dar la mejor respuesta a las necesidades de todo el alumnado. Este argumento es reiterado y utilizado por todo el profesorado del centro, quienes asumen que la utilización de esta estrategia organizativa responde a las buenas intenciones de la institución y a su compromiso por hacer todo lo que esté en sus manos para fomentar el aprendizaje de todo el alumnado:

"No me puede a mi convencer nadie de que en una clase donde hay alumnos de todo, con el criterio de que no se tienen que seleccionar los alumnos, que esto es bueno para ellos. Porque esto es absurdo completamente [...] ¿Lo importante qué es? Que aprendan. Eso es lo fundamental: que utilicemos todas las herramientas y todos los recursos para que ese alumno llegue a eso que llamamos mínimos". ( $\mathrm{M}^{\mathrm{a}}$ Ángeles, tutora de $3^{\circ} \mathrm{B}$ y profesora de Ciencias Sociales, Instituto del Canal)

El argumento de que esta estrategia se desarrolla desde "la buena voluntad" y pensando en "lo mejor" para todo el alumnado resultaría, en principio, difícilmente objetable. Y más cuando se constata que el número de alumnos disminuye a medida que el "nivel es más bajo", una situación que podría entenderse como el reconocimiento de una necesidad de atención mayor hacia el alumnado de estos grupos. Ahora bien, las prácticas que en los diversos agrupamientos acontecen, como después abordaremos, no corroboran los objetivos iniciales que se explicitan y enmascaran las concepciones diversas, y jerarquizadas, que el profesorado tiene respecto a las posibilidades del logro escolar de los estudiantes, y a sus capacidades, en cada uno de los agrupamientos.

\section{b) La eficiencia: ¿aprovechar los recursos limitados?}

El segundo argumento que se utiliza de manera explícita es que el agrupamiento por niveles es una estrategia adecuada en términos de eficiencia, convirtiéndose en la mejor forma de aprovechar los recursos disponibles. De hecho, el propio director explica que utilizar un sistema de agrupamiento heterogéneo supondría un desperdicio de recursos y una peor atención al alumnado. Por otro lado, el profesorado alude a las dificultades vinculadas a la escasez de recursos, principalmente humanos, haciendo un énfasis específico en las altas ratios: “¡Hay muy pocos recursos! Tú no puedes hacer grupos de 30 tan heterogéneos. Es perfecto cuando son 15 o $20 \ldots$ Pero con 30 no se puede atender a la diversidad". (Verónica, profesora de matemáticas, Instituto del Canal)

Si bien hay que reconocer que los recursos de los centros educativos son limitados y a menudo insuficientes, siendo ésta la argumentación que ofrece el profesorado, éste parece no resultar ser el motivo real que explica la opción organizativa. De hecho, a lo largo del trabajo de campo, se preguntó de forma reiterada sobre el supuesto impacto que podría tener un aumento de los recursos del centro en la mejora de las condiciones de escolarización del alumnado. Aunque de forma unánime todo el profesorado mostró su acuerdo en que un incremento de recursos (humanos, principalmente) podría suponer mejoras en el centro, este impacto se subestimó de forma recurrente:

\footnotetext{
Alba: Si el centro tuviese más recursos, ¿crees que...?

Carme: No creo que sean recursos.

Alba: ¿No?

Carme: No. Bueno, evidentemente, cuanta más gente hay... Pero a veces no son tanto los recursos sino la capacidad de poder hacer llegar el mensaje. Y a menudo, no podemos hacerlo llegar, no por falta de personas, sino porque el receptor no quiere. Puedes tener, en lugar de un tutor por grupo, tener dos. Es igual. [...] La cuestión es que el mensaje no llega porque el receptor no quiere. (Carme, profesora de Física y Química, Instituto del Canal)
}

\subsection{Dos realidades sociales, dos realidades académicas: la inviabilidad de los espacios compartidos}

Un tercer argumento, identificado tanto en los discursos del equipo directivo como del profesorado, es la incapacidad del instituto para proporcionar una enseñanza adecuada y satisfactoria, para todo el alumnado, de una manera alternativa al agrupamiento por niveles. Es decir, el agrupamiento por niveles es visto como la única opción viable para conseguir una 
enseñanza eficaz:

"Yo no digo que sea lo mejor [agrupar por niveles], de acuerdo. Pero si estuviésemos en Sarriá [un barrio de Barcelona caracterizado por su alto nivel socioeconómico], esto no haría falta. Pero claro, con el panorama que tenemos, si no separas es imposible". (Natasha, profesora de Lengua Inglesa, Instituto del Canal. Diario de campo, 27 de mayo del 2015)

La composición social del instituto emerge como una de las razones por las cuales se justifica que no es "posible" ni "viable" utilizar otra estrategia de agrupamiento. El fundamento de este argumento es que el propio alumnado, al ser tan "diverso" o "heterogéneo", impide que los procesos de aprendizaje puedan desarrollarse en grupos heterogéneos. Como señala esta profesora, frente a las dificultades que representaría tener que enseñar a una población escolar "tan" diversa, el agrupamiento por niveles aparece como la solución para reducir el "rango de diversidad": "Si con grupos de 20 no se puede atender a la diversidad, la única solución es que la diversidad desaparezca”. (Verónica, profesora de matemáticas, Instituto del Canal)

Así, desde el Instituto del Canal se asume que las desigualdades sociales generan unas diferencias académicas que convierten al alumnado en muy "diverso" y con necesidades altamente divergentes, dando por sentado, por tanto, que "es imposible" enseñarles en espacios compartidos.

A continuación se exploran los criterios de separación que se utilizan para clasificar al alumnado en los grupos, con el objetivo de "reducir" la diversidad existente.

\subsection{La clasificación del alumnado: la primacía del mérito}

Los criterios de distribución de los estudiantes en los grupos son un elemento fundamental a la hora de analizar esta estrategia pues, más allá de poder comprender el propio proceso de "clasificación" del alumnado, permiten identificar las lógicas subyacentes al propio modelo de agrupamiento. Por estas razones, se analizan a continuación los criterios de clasificación del alumnado empezando por los que se declaran desde el discurso institucional y explorando, después, otros criterios que emergen a la hora de hacer la separación efectiva.

\section{a) El criterio declarado: los ritmos de aprendizaje}

Desde el discurso institucional del Instituto del Canal se señala que las variables para confeccionar los grupos responden a cuestiones académicas, esto es, vinculadas con el aprendizaje formal, los conocimientos y las competencias que la escuela quiere trasmitir. De hecho, si la pretendida finalidad del agrupamiento por niveles es, como argumenta el profesorado, proporcionar una enseñanza adecuada a todo el alumnado, parecería razonable que los criterios de selección se basaran en las necesidades académicas de éstos. En este sentido, el criterio de distribución al que se alude en la documentación y en los discursos oficiales es el «ritmo de aprendizaje» del alumnado:

\footnotetext{
"Intentamos hacer dos niveles, por decirlo de alguna manera. En el primer ciclo [de la ESO], el A y el $\mathrm{B}$, con un nivel, el $\mathrm{C}$ y el $\mathrm{D}$, con otro. O sea, dos ritmos de aprendizaje. Hablamos de ritmos de aprendizaje que queda más... Si hablamos de ritmos de aprendizaje, pues hay un ritmo de aprendizaje más rápido, y hay un ritmo de aprendizaje más lento". (Pepe, Director, Instituto del Canal)

"El criterio que utiliza nuestro centro para elaborar las distintas agrupaciones es teniendo en cuenta el ritmo de aprendizaje del alumnado. Las agrupaciones son flexibles, de forma que se pueden hacer cambios preferentemente hasta la $1^{\mathrm{a}}$ evaluación”. (Proyecto Educativo de Centro, Instituto del Canal, p. 9)
}

Si bien el "ritmo de aprendizaje", la "velocidad" a la que una persona aprende, es el criterio que se menciona cuando se habla de la clasificación del alumnado, no existe ninguna otra referencia en el PEC, ni en otro documento, sobre qué se entiende, en el Instituto del Canal, por "ritmo de aprendizaje", ni cómo se debería medir o evaluar. Sin embargo, por el uso que se hace de este concepto en los discursos habituales del profesorado, se infiere que éste hace referencia a las 
"capacidades" o a las "habilidades innatas" del alumnado. Esta inferencia se puede hacer de expresiones como las siguientes: "Hay niños que les cuesta mucho seguir el ritmo, y hay otros que tiran solos, que ya se ve que tienen más capacidades". (Elena, Coordinadora Pedagógica, Instituto del Canal). "Hay chicos que marchan más autónomamente, que tienen más facilidad, y otros que necesitan una atención más directa del profesor porque les cuesta mucho". (Pepe, Director, Instituto del Canal)

En estos fragmentos se evidencia que el "ritmo de aprendizaje" es considerado una característica que refleja las capacidades del alumnado ("tiran solos", "tienen facilidad", "les cuesta mucho"), asumiendo que éstas son un atributo fijo y estable, y que se pueden determinar de forma precisa. Sin embargo, la ambigüedad del constructo "ritmos de aprendizaje", y la inexistencia de indicadores concretos y de criterios explícitos para medirlos y evaluarlos, hace que otros elementos emerjan como variables de clasificación.

\section{b) Los criterios tácitos: actitudes, bábitos y comportamiento}

Como ha mostrado de forma extensa la investigación educativa, los elementos actitudinales (por ejemplo, los hábitos de estudio, la disciplina y el interés percibido hacia la escuela y la educación) son un factor central en la valoración que el profesorado hace de su alumnado. Estos elementos adquieren trascendencia, también, en el Instituto del Canal a la hora de clasificar al alumnado en los grupos, aunque en los documentos institucionales no se les dote de tal entidad.

La importancia de las actitudes en los procesos de selección del alumnado es un elemento que se percibe de manera evidente cuando se observa la vida del centro y se conversa con el profesorado y los y las jóvenes. Si bien, en un plano institucional, se defiende que el alumnado se debería separar según sus "ritmos de aprendizaje", hay numerosos ejemplos que constatan que este criterio y sus distintas aproximaciones no son siempre los factores determinantes para su asignación en los grupos:

"Normalmente tú tienes un grupo A y, en principio, la conducta es correcta porque son niños con hábitos, sobre todo con hábitos de trabajo. Representa, representa, que están separados por hábitos de trabajo. Entonces, va más o menos ligado, los hábitos de trabajo con la actitud mala: menos hábitos de trabajo, entonces más distracción, entonces menos concentración... Por fuerza se tienen que distraer, y la conducta se ve afectada”. (Carme, profesora de Física y Química, Instituto del Canal)

En definitiva, los grupos en el Instituto del Canal se conforman principalmente según el grado de cumplimiento de las "obligaciones" que los jóvenes tendrían que satisfacer por ser estudiantes, esto es, hacer los deberes, tener hábitos de estudio y de trabajo, esforzarse y mostrar interés en su aprendizaje. De esta manera, el grupo A está conformado por los alumnos que "hacen lo que hay que hacer", mientras que en el grupo C se asigna a los alumnos que no cumplen con estos compromisos.

La materialización más evidente de que satisfacer las demandas de la institución escolar es un criterio de clasificación, se comprende en el momento en que se exploran los motivos por los cuales hay alumnos que, "a pesar" de su "ritmo de aprendizaje" o de sus "capacidades", están en un grupo que no les correspondería de acuerdo con estos criterios. Hay dos situaciones que evidencian este hecho: el caso de las llamadas por el profesorado "las chicas 'justitas', pero que trabajan mucho", y que están en el grupo A; y el caso de los definidos como "los 'elementos' conflictivos, que son listos pero no quieren hacer nada", que están en el grupo C. Si en el caso de las "chicas trabajadoras pero limitadas", "las justitas", se recompensa su esfuerzo y el cumplimiento con las tareas y compromisos escolares mediante las notas y su posición en el grupo A, en el caso de los "chicos listos pero disruptivos" se sanciona su actitud considerada conflictiva y desafiante situándoles en el grupo C, una situación que por otra parte añade una mayor complejidad al grupo. El valor otorgado a estos factores actitudinales pone de manifiesto que, en los procesos de clasificación del alumnado, se prescinde de los elementos rigurosamente académicos anteponiendo los elementos expresivos vinculados a las expectativas de la institución escolar. 


\subsection{La puesta en práctica del modelo: segregación social y oportunidades de aprendizaje desiguales}

\section{a) La composición social de los grupos}

Después de lo presentado hasta ahora, es relevante mostrar la composición de los grupos, en términos socioeconómicos y culturales, para ver de qué manera los criterios utilizados para distribuir al alumnado en los grupos condicionan su composición. En efecto, los datos recogidos durante el trabajo de campo confirman que existe una distribución desigual del alumnado en los grupos teniendo en cuenta, precisamente, los factores socioeconómicos y culturales de sus familias.

En términos de nivel educativo de los progenitores, se observan diferencias relevantes entre los diversos grupos de $3^{\circ}$ y $4^{\circ}$ de la ESO. Como muestra la Tabla 2, a medida que "disminuye el nivel" de los grupos, disminuyen también las familias con un capital instructivo elevado, y aumentan las familias con un nivel de instrucción bajo. Así, mientras que en el grupo $C$ hay un $30 \%$ de chicos y chicas cuyos progenitores han estudiado, como máximo, hasta la educación primaria, este porcentaje es solo del $8 \%$ en el caso del grupo A.

Tabla 2

Nivel de estudios más alto de los progenitores, distribución por grupos

\begin{tabular}{lccc}
\hline & Grupo A & Grupo B & Grupo C \\
\hline Educación primaria o menos & $8 \%$ & $24 \%$ & $30 \%$ \\
Educación secundaria & $28 \%$ & $22 \%$ & $20 \%$ \\
Ciclos Formativos o Bachillerato & $30 \%$ & $22 \%$ & $33 \%$ \\
Universidad & $18 \%$ & $16 \%$ & $3 \%$ \\
No sabe & $15 \%$ & $16 \%$ & $13 \%$ \\
\hline
\end{tabular}

Fuente: elaboración propia a partir del cuestionario del alumnado de $3^{\circ}$ y $4^{\circ}$ de la ESO

Sin embargo, las diferencias sociales más acusadas entre los grupos son las que se observan según el estatus migrante del alumnado, tal y como se muestra en la Tabla 3. Mientras que el grupo A está compuesto, de forma mayoritaria, por alumnado autóctono (éstos representan casi el $80 \%$ del grupo), en el grupo C esta proporción se reduce a la mitad, al 40\%. Obviamente, esta relación se invierte en el caso del alumnado inmigrante, que en el grupo $\mathrm{C}$ representa el $60 \% \mathrm{y}$, en el grupo A, tan sólo el 20\%. Más allá del estatus migrante del alumnado, se observan también diferencias severas en cuanto a la región de procedencia del alumnado que ha nacido fuera del territorio español (también en la Tabla 3). En este sentido, es destacable que en los grupos A y B, la mayor parte del alumnado inmigrante es de origen latinoamericano, mientras que en el grupo $\mathrm{C}$ son jóvenes marroquíes.

Tabla 3

Estatus migrante y región de procedencia del alumnado inmigrante, distribución por grupos. Instituto del Canal

\begin{tabular}{lllll}
\hline & & Grupo & Grupo & Grupo \\
& & A & B & C \\
\hline Estatus migrante & Autóctonos & $79 \%$ & $64 \%$ & $39 \%$ \\
& Inmigrantes de primera & $19 \%$ & $33 \%$ & $58 \%$ \\
& generación & & & \\
& Inmigrantes de segunda & $2 \%$ & $3 \%$ & $3 \%$ \\
Región de procedencia alumnado & generación & & & \\
inmigrante & Latinoamérica & $85 \%$ & $77 \%$ & $22 \%$ \\
& Magreb & $7 \%$ & $8 \%$ & $64 \%$ \\
\hline
\end{tabular}

Fuente: elaboración propia a partir del cuestionario del alumnado de $3^{\circ}$ y $4^{\circ}$ de la ESO.

Los datos presentados, pues, muestran que existe una distribución desigual entre los grupos según las características socioeconómicas y culturales de las familias, hecho que corrobora que, 
efectivamente, el agrupamiento por niveles se utiliza para que "dos realidades sociales diversas" no se mezclen. Esto muestra, en definitiva, que los criterios utilizados para clasificar al alumnado en los grupos (esto es, los "ritmos de aprendizaje", el "rendimiento académico" y las "actitudes") no son neutros social y culturalmente: la distribución del alumnado hace evidente que el alumnado que proviene de familias con mayor capital instructivo pero, sobre todo, de familias autóctonas, se ve favorecido a la hora de ser asignado en los grupos de nivel "altos".

\section{b) Prácticas curriculares y de instrucción}

A pesar de que en Cataluña exista un currículum oficial que dictamina cuales deben ser los contenidos y competencias básicas que todo el alumnado debe alcanzar al final de la educación obligatoria, la puesta en práctica de este currículum básico difiere según los contextos y el alumnado al que se haga referencia, tal y como se ha podido constatar a lo largo de esta investigación. Mediante un proceso de reelaboración los docentes adaptan el currículo oficial, y modifican los contenidos y las competencias que se trabajan en las aulas. El propósito de este subapartado es explorar, a la luz de los datos recogidos, el currículum "de facto" (Anyon, 1981) que se imparte en los diversos agrupamientos, eso es, las formas de concreción curricular e instructiva, mostrando las variables que explicarían las diferencias efectivas en dicho ejercicio de concreción.

Un primer elemento que se debe desatacar es que la propia constitución de grupos diferenciados tiene una influencia fundamental en la configuración de las expectativas y creencias docentes, que son altamente dispares entre los grupos, y que tienen una influencia crucial en la concreción curricular e instructiva. Según se desprende del análisis realizado, estas expectativas y creencias desiguales se sustentan, por un lado, en las diferencias socioeconómicas y culturales del alumnado de cada uno de los grupos, pero, sobre todo, se ven reforzadas por el significado tácito que adquieren los grupos, no únicamente en términos de "capacidades" y hábitos escolares, sino también en términos de intereses, motivaciones y trayectorias futuras. En este sentido, más allá de la construcción de la capacidad / incapacidad para adquirir los contenidos de la educación obligatoria, la conjunción entre las características sociales, étnicas y culturales del alumnado y el significado tácito de los grupos de nivel inciden también en la construcción de un imaginario docente acerca de su futuro académico, laboral y vital, que condiciona las consideraciones sobre qué competencias y conocimientos necesitan estos alumnos para su futuro y, por lo tanto, sobre qué se les debería enseñar.

Así pues, en el Instituto del Canal, la organización del alumnado en grupos de nivel implica una diferenciación significativa en cuanto a los contenidos trabajados: existen importantes diferencias entre grupos en cuanto al currículum y el tipo de competencias y habilidades que se fomentan, diferencias que se repiten en las distintas materias, tal y como se explora a continuación.

En primer lugar, esta diferenciación curricular radica en una rebaja importante de los objetivos de aprendizaje del grupo C mientras que en el grupo A (y en la mayoría de los casos también en el grupo B) los contenidos trabajados se ajustan al temario propuesto en el currículum oficial. Esta rebaja en los grupos de nivel "bajos", como se desprende de las palabras del profesor de catalán, no es "oficial", sino que se desarrolla bajo unos parámetros supuestamente ordinarios:

"El libro, la programación del A y del B, con chavales del C es imposible hacerlo. Aunque oficialmente tenemos una programación y deben hacer aquello, los del C no lo hacen". (Francesc, tutor de $3^{\circ}$ A y profesor de Lengua Catalana, Instituto del Canal)

"Yo no había hecho exactamente lo mismo en el $3^{\circ} \mathrm{A}$ y en el $3^{\circ} \mathrm{B}$, pero en el momento de empezar el tema de literatura medieval, pensé: «voy a probar de hacerlo exactamente igual en el A y en el B, a ver si son capaces de seguir la explicación». Y bueno, he visto que sí, que la han seguido, e incluso me han hecho preguntas interesantes [...]. Y bueno, con el $\mathrm{C}$ ya ni me lo planteo, porque llegamos al nivel de que no saben ni el presente de indicativo del verbo cantar [...]. Con estos [el grupo de $3^{\circ} \mathrm{C}$ ] estoy haciendo cosas básicas. O sea, ahora se están aprendiendo los verbos. O sea, es que no saben diferenciar el futuro del pretérito perfecto. Les suena a chino". (Carolina, profesora de Lengua Castellana en $3^{\circ} \mathrm{A}, \mathrm{B}$ y C, Instituto del Canal) 
Esta rebaja de las exigencias académicas se materializa en la profundidad de los contenidos trabajados en cada grupo. Mientras que en los grupos A y B los materiales utilizados responden al currículum ordinario -aunque el ritmo de enseñanza pueda diferir-, en el grupo $\mathrm{C}$ se utiliza un material de adaptación que supone trabajar los contenidos oficiales de forma simplificada, reducida y más superficial:

\begin{abstract}
"Cuando la profesora a la que substituyo me dio las indicaciones por escrito, pues me dijo que los de $3^{\circ} \mathrm{B}$ iban más lentos que los del $\mathrm{A}$, pero que seguían el mismo libro con un ritmo bastante más pausado. Con los del $3^{\circ} \mathrm{C}$ ya me dijo que era otro tipo de libro totalmente diferente, que les costaba mucho, y que, bueno, que lo que más hacía era vocabulario, y que el libro es como de adaptación curricular". (Carolina, profesora de Lengua Castellana en $3^{\circ}$ A, B y C, Instituto del Canal)
\end{abstract}

Teniendo en cuenta que la cobertura del currículum es incompleta en el grupo C (en el A y B, aunque no se logre cubrir toda la extensión del currículum, el profesorado no alude a esta situación), existe una selección y priorización del temario para ajustar el tiempo y los esfuerzos a los mínimos que se deben intentar cubrir, tal y como explicaba la profesora de castellano en un fragmento anterior. En este sentido, el profesorado admite que, en la docencia de los grupos de nivel "bajos", se modifica el currículum reduciendo los contenidos y las competencias que el alumnado debería aprender y desarrollar, omitiendo aquellas "cosas que no son imprescindibles":

\begin{abstract}
"Ya ves lo que hemos hecho hoy en clase de $3^{\circ} \mathrm{C}$ : trabajar las funciones a partir de una factura de teléfono, pero ni eso entendían. Por eso, yo en este grupo [se refiere a $3^{\circ} \mathrm{C}$ ] intento que tengan lo básico por si alguien pasa a $4^{\circ}$, que tenga los conocimientos básicos de las funciones. Pero poco más puedo hacer. En cambio, en el A, doy el temario normal, y en el B también pero un poco menos". (Verónica, profesora de matemáticas, Instituto del Canal)

"En el $3^{\circ} \mathrm{C}$ utilizo un libro de $2^{\circ}$ de la ESO adaptado. Y hasta hay partes que me las salto, porque son cosas que no son imprescindibles. Trabajar la poesía y los poemas, por ejemplo, no es imprescindible y tampoco quiero desmotivarles con cosas muy difíciles [...]. Con ellos $\left[3^{\circ} \mathrm{C}\right]$ trabajo básicamente ortografía y comprensión lectora. Lo básico. Redacciones no, porque es un desastre [...]. Y muchas veces, me preguntan para qué sirve lo que les explico. Porque en su entorno directo no escuchan ni una palabra de catalán, nunca. Entonces, claro, me preguntan por qué tienen que saber combinar los pronombres débiles". (Alicia, profesora de Lengua Inglesa pero que imparte Lengua Catalana en $3^{\circ}$ de la ESO C, Instituto del Canal).
\end{abstract}

En el conjunto de fragmentos anteriores se identifican diferentes tipos de argumentos que el profesorado utiliza para justificar la selección reducida de los contenidos en el grupo C. El primero de ellos es que las capacidades del alumnado de este grupo no serían suficientes para alcanzar el contenido general, ya que "no llegan a más", "no saben suficiente" o "no entienden nada". Por otro lado, vinculado con las expectativas de los docentes en relación al futuro académico, laboral y social de estos estudiantes, la selección de los contenidos se hace en relación a la utilidad otorgada a dichos contenidos. Como se ha visto, se hace referencia, por un lado, a proporcionar los mínimos para que estos chicos y chicas continúen su formación, aunque las expectativas de continuidad -y la confianza en que esto se dé- son muy reducidas ("por si alguien pasa a $4^{\circ}$ "); y a "recortar" los contenidos prescindibles, aquellos que el alumnado del grupo C "no necesita", esto es, aspectos más complejos que sí deben aprender los del grupo A y B (por ejemplo los pronombres débiles a los que hacía referencia la profesora de catalán). En este sentido, el currículum se concibe de forma distinta en función del grupo: para el grupo A se trata de un currículum que les debe servir para "saber cosas, tener un bagaje como persona, y para seguir estudiando". En cambio, para otros alumnos (del grupo C) se reduce la utilidad del currículum hasta concretarse en funciones básicas, "de supervivencia".

La diferenciación de los objetivos de aprendizaje se traslada, obviamente, a las actividades diseñadas para desarrollar las competencias y habilidades adecuadas a cada uno de los grupos. El "buen" comportamiento del alumnado del grupo A promueve que el profesorado dedique más tiempo al diálogo, a la exposición magistral y al desarrollo de actividades intelectuales "más elevadas" 
mientras que en el grupo C, la asignación de tareas individuales se utiliza para gestionar el control del aula, a través del logro del silencio.

\title{
4.4. Como se vive el instituto en cada grupo
}

Un último elemento que abordamos es la experiencia escolar del alumnado, mostrando las vivencias y percepciones de los y las jóvenes del grupo A (de "alto" nivel), de aquellos y aquellas del grupo C (el grupo de "bajo" nivel), ya que ofrecen un mayor contraste y permiten identificar de forma más clara las consecuencias que para la experiencia escolar tiene el agrupamiento por niveles.

\section{a) El grupo A: estar en la cumbre de la jerarquía escolar}

Los y las jóvenes del grupo A tienen una alta autoestima académica y una elevada seguridad en sus posibilidades. El hecho de estar en el grupo A les posiciona en el eslabón superior de la jerarquía escolar y esto, obviamente, tiene repercusiones en cómo se sienten y cómo afrontan su día a día. En este sentido, estos y estas jóvenes afirman tener las "capacidades" necesarias para estar en el grupo más alto, así como tener un interés suficiente para merecer estar en este grupo. De hecho, al ser preguntados por los motivos que explican su lugar en el grupo A todos y todas aluden, de forma casi unánime, a los mismos factores:

Alba: ¿Y tú por qué crees que estás en este grupo?

Júlia: No sé [se ríe]. Yo creo que porque aprendo rápido, supongo, y porque yo realmente tengo interés en aprender y aprobar, esto sobretodo. (Júlia, alumna de $3^{\circ} \mathrm{A}$, Instituto del Canal)

Esta interiorización de la capacidad es especialmente visible entre el alumnado que, a lo largo de su trayectoria educativa, ha conseguido "escalar posiciones" en la jerarquía escolar, como muestra el ejemplo de Maryam:

\author{
Alba: ¿Y tú por qué estás en el A? \\ Maryam: No lo sé. Yo vine de la B \\ Alba: ¿Y por qué te cambiaron? \\ Maryam: No sé, yo me esforzaba mucho, por eso, por el esfuerzo. A ver, que si no me hubiesen visto \\ con capacidad, supongo que no me hubiesen cambiado, claro. (Maryam, alumna de $3^{\circ} \mathrm{A}$, Instituto del \\ Canal)
}

Estas citas representan cómo el alumnado del grupo A se "autoconstruye" y se "autopercibe": el elemento esencial es que, además de la voluntad y el interés en aprender y aprobar, el alumnado del grupo A se siente capaz, cree que es apto para asumir el nivel académico exigido y salir adelante satisfactoriamente. Así, para el alumnado del grupo A, estar en este grupo representa un reconocimiento merecido, por parte de la institución escolar, de su esfuerzo e interés, pero también de sus capacidades.

\section{b) El grupo C: ser de la parte baja de la jerarquía escolar}

El grupo C está conformado por aquellos y aquellas estudiantes que "requieren una atención específica" y es concebido, por parte del profesorado y de la institución, como el más lejano a la idea del "buen alumno". Esta construcción, y la valoración de la misma, inciden de forma incuestionable en las experiencias de los chicos y chicas del grupo C, opuestas a las de sus compañeros y compañeras de los otros grupos.

Las percepciones que los y las jóvenes del grupo C tienen acerca de las expectativas de su profesorado son especialmente cruentas y demoledoras. Estas percepciones, que se derivan de cómo el profesorado interacciona con el grupo, cuestionan las "capacidades" del alumnado, y también sus posibilidades de futuro:

Alba: ¿Y tú qué crees que piensan los profes de vosotros? [silencio]

Shaima: Que la liamos mucho... [silencio]. Yo qué sé, profe... [su tono expresa resignación]. A lo 
mejor piensan que somos tontos. (Shaima, alumna de $3^{\circ} \mathrm{C}$, Instituto del Canal)

Hay profes que a veces nos han dicho a la C, algunos, eh?, no todos. Han dicho: «si vais a estar aquí [en la C] no os vais a sacar nada», y pues eso también... jvaya ánimos! (Ester, alumna de $3^{\circ} \mathrm{C}$, Instituto del Canal)

Además, el alumnado del grupo C también parece ser consciente de que su profesorado pone en cuestión, no solamente sus capacidades, sino también su integridad moral y su valor como personas:

Alba: ¿Y tú qué crees que me dirían los profes de vosotros si yo fuese una profesora nueva?

Jamila: Pues que somos malos, que somos unas bestias [se ríe, nerviosa]. (Jamila, alumna de $3^{\circ} \mathrm{C}$, Instituto del Canal)

Alba: ¿Y tú qué crees que piensan los profes de vosotros?

Yassin: Se piensan que somos animales.

Alba: ¿Cómo que se piensan que sois animales?

Yassin: Mmm... Piensan que cualquier profe que va a entrar allí se va a volver loco o algo. El otro día estaba en prefectura, y había muchos profes, y nosotros no teníamos profe. Y le dije al director: «¿puede ir alguna profe que suba con nosotros a clase?» Y ninguno ha querido subir. Ninguno. (Yassin, alumno de $3^{\circ} \mathrm{C}$, Instituto del Canal)

Por último, las bajas expectativas que tiene el profesorado acerca del alumnado de este grupo se manifiestan en las formas en que el profesorado se dirige al grupo, en cómo plantea su relación con estos estudiantes, y cómo reacciona ante las respuestas -a veces desafiantes, a veces de indiferencia- que tiene el alumnado del grupo $C$ para con el aprendizaje y el instituto:

Eva: Pues todos los profes, todo el mundo, cree que somos un desastre.

Alba: ¿Cómo sabes que es así? ¿Por qué lo dices?

Eva: Porque se les nota en las caras, a algunos profes. No con todos se portan igual, pero con algunos que se portan más o menos mal, se les nota en la cara. Que no tienen ganas ni de venir aquí a esta clase.

Alba: ¿Tú crees que hay profes que no quieren venir a esta clase?

Eva: Sí. Muchos. (Eva, alumna de $3^{\circ} \mathrm{C}$, Instituto del Canal)

Alba: Y los profes ¿qué crees que piensan de vosotros?

Alex: Hay algunos que bueno, que nos tienen menos aprecio que otros. Porque hay algunos que no, que no quieren ni hacer clase aquí, que no nos soportan. Esto se nota. Es que a veces se ve en la cara, ya. Se ve con las ganas que entran, ya se nota. (Alex, alumno de $3^{\circ} \mathrm{C}$, Instituto del Canal)

Los fragmentos anteriores expresan con dureza las percepciones que tiene el alumnado del grupo $\mathrm{C}$ acerca de las creencias de sus docentes. En primer lugar, el alumnado destaca el rechazo que perciben de la mayoría de su profesorado ("no quieren hacer clase aquí", "se les ve en la cara", "ninguno ha querido subir", "no nos soportan"). En segundo lugar, el alumnado también percibe una minusvaloración de sus capacidades ("piensan que somos tontos", "nos dicen que no nos vamos a sacar nada"). Y en tercer lugar, señalan una estigmatización por su comportamiento ("se piensan que somos animales", "te dirían que somos unas bestias"). Obviamente, la congruencia en las interpretaciones que hace el alumnado sobre los pensamientos y expectativas de sus docentes obedece a que el profesorado expresa un conjunto de mensajes coherentes entre sí que trasladan estas percepciones al alumnado, tales como las malas caras cuando entran en clase, comentarios despectivos reiterados, etc.

Por último, un factor importante que señalan de forma unánime los y las jóvenes del grupo $\mathrm{C}$ es precisamente el impacto del grupo en sus actitudes y comportamientos. Si en el caso del grupo A se identifica la presión grupal como un estímulo corrector para amoldarse a las exigencias escolares, en el caso del grupo $\mathrm{C}$ parece que existe un efecto inverso, en que los y las jóvenes destacan el "efecto contagio" que les supone estar en este grupo. Pero no solamente esto, sino que el alumnado del grupo "bajo" reconoce que, en buena parte, su conducta se explica cómo "efecto espejo" por las injusticias y el desprecio percibido por parte del profesorado. Asimismo, los impactos de estar en el grupo $\mathrm{C}$ van más allá de la experiencia actual del alumnado, y también condicionan su futuro: el significado que adquiere "ser" del grupo C limita y restringe los imaginarios de los chicos y chicas en cuanto a la educación postobligatoria, pero también sus posibilidades instrumentales a nivel de 
adquisición de competencias y habilidades, tal y como reconocen los propios jóvenes.

\section{Conclusiones}

El interés de este artículo ha sido explorar las dinámicas escolares que se desarrollan en la educación secundaria obligatoria en entornos de alta complejidad social y comprender cómo se configuran las oportunidades y las experiencias del alumnado en dichos contextos. Concretamente y mediante una metodología etnográfica, se han analizado las prácticas de agrupamiento del alumnado y las formas en que estas prácticas, sujetas a las creencias y expectativas docentes, condicionan las experiencias de los y las jóvenes.

El modelo de agrupamientos homogéneos se basa en un proyecto estructurado a partir de asunciones tácitas que no están escritas, pero que son las que en realidad rigen el funcionamiento de los grupos. Si bien la clasificación del alumnado se hace, formalmente, en base a sus "ritmos de aprendizaje", los criterios efectivos de clasificación son el rendimiento académico y las disposiciones expresivas del alumnado acerca de la educación (hábitos de estudio y esfuerzo). Este segundo criterio establece una distinción entre el buen alumno (el que se esfuerza y que tiene hábitos) y el mal alumno (el que no cumple con estas disposiciones), que refleja, también, una distinción del alumnado según su origen étnico y social. En este sentido, el uso de las disposiciones como criterio de selección privilegia al alumnado más aventajado socialmente, que se concentra en los grupos de nivel "alto", mientras que sanciona -mediante su asignación a los grupos "bajos"- al alumnado más alejado de la norma escolar y más lejano étnica y socialmente del "alumno ideal", típicamente de clase media (Becker, 1952). Se puede afirmar que, más allá de los argumentos legitimadores explicitados en los discursos institucionales, el agrupamiento por niveles se articula alrededor de un sistema de "recompensa-sanción" que premia a aquellos y aquellas estudiantes que encajan con la imagen de alumno ideal, mientras que sancionan a aquellos que se alejan de este modelo.

Esto se refleja en la composición social y académica de los grupos. Mientras que en los grupos "altos" el alumnado proviene de entornos familiares más aventajados socioeconómicamente y de familias principalmente autóctonas, en los grupos "bajos" existe una concentración de alumnado extranjero, y de familias con menor capital instructivo. Esto muestra que los criterios utilizados para clasificar al alumnado en los grupos no son neutros social y culturalmente. Esta distinción, así como el significado simbólico que adquieren los grupos, comporta una construcción de expectativas docentes muy diferenciadas que repercuten en los procesos de enseñanza-aprendizaje y en las experiencias de los jóvenes.

Las creencias y expectativas sostenidas sobre la población escolar -basadas en estereotipos acerca del alumnado más desfavorecido y sus familias- emergen como una justificación para el agrupamiento homogéneo, que se utiliza con el objetivo de reducir el rango de diversidad percibida. Asimismo, la propia constitución de grupos diferenciados tiene una influencia fundamental en la configuración de las expectativas y creencias docentes, que son altamente dispares entre los grupos. Según se desprende del análisis realizado, estas expectativas y creencias desiguales se sustentan, por un lado, en las diferencias socioeconómicas y culturales del alumnado de cada uno de los grupos, pero, sobre todo, se ven reforzadas por el significado tácito que adquieren los grupos, no únicamente en términos de "capacidades" y hábitos escolares, sino también en términos de intereses, motivaciones y trayectorias futuras. En este sentido, se ha detectado un "efecto de categorización" (Kelly y Carbonaro, 2012), es decir, que la información que las "etiquetas institucionales" proporcionan al profesorado son un factor clave para comprender la configuración de las expectativas diferenciadas, que modelan, a su vez, las posibilidades de acción percibidas por parte del cuerpo docente.

Ahora bien, frente el agrupamiento homogéneo y sus resultados, no se observa un cuestionamiento del modelo en sí, ni un análisis de las condiciones que desde una perspectiva inclusiva el centro ofrece (Booth y Ainscow, 2002) como tampoco se percibe la búsqueda de nuevas propuestas metodológicas que representen su superación, sino que las críticas que puedan existir al 
modelo se centran mayormente en disfunciones o contradicciones derivadas de su aplicación. Son pocos los profesionales que destacan la injusticia que este sistema puede suponer para el alumnado de los grupos "bajos". Resulta evidente la limitación de oportunidades a la que se ve sometido el alumnado de estos grupos, tanto a nivel instrumental (la no adquisición de las competencias básicas les sitúa en desventaja a la hora de cursar estudios postobligatorios) como a nivel de imaginario futuro (el agrupamiento "encasilla" prematuramente al alumnado de los grupos "bajos" reduciendo sus aspiraciones y expectativas académicas posteriores).

La naturalización de los procesos y resultados educativos es habitual en las instituciones escolares, al ser parte de la red de interpretaciones compartidas que la propia institución tiende a promover. El estudio de la cultura y la estructura de la institución escolar desde una lógica etnográfica ha posibilitado identificar estos procesos cotidianos de naturalización de las desigualdades al permitirnos conocer las formas, mecanismos y dinámicas que otorgan condiciones desiguales a los jóvenes al situarlos en espacios diferenciados a través de los agrupamientos de nivel.

Todas las figuras deberían numerarse con números arábigos (1., 2., 3.,...). Cada figura debe tener título. Todas las fotografías, esquemas, gráficos y diagramas deben estar citados en el texto como figuras. Los dibujos lineales deberán tener buena calidad, tanto si son escaneados como si se trata de originales generados electrónicamente. El material escaneado de baja calidad no será aceptado. Las figuras deben incluirse en el texto y no entregarse por separado. En el archivo de origen MS Word las figuras deben ir convenientemente codificadas. Las letras y/o los símbolos deben estar claramente definidos en cada título o en la leyenda facilitada como parte de la figura. Las figuras deben colocarse en la parte superior o inferior de la página, lo más cerca posible de la primera cita en el artículo.

\section{Referencias}

Abadzi, H. (1985). Ability Grouping Effects on Academic Achievement and Self-Esteem Who Performs in the Long Run as Expected. Journal of Educational Research, 79(1), pp. 36-40. doi: 10.1080/00220671.1985.10885644.

Anyon, J. (1981). Social Class and School Knowledge. Curriculum Inquiry, 11(1), pp. 3-42. doi: 10.1080/03626784.1981.11075236.

Becker, H. S. (1952). Social-Class Variations in the Teacher-Pupil Relationship. Journal of Educational Sociology, 25(8), pp. 451-465. doi: 10.2307/2263957.

Booth, T., \& Ainscow, M. (2002). Index for Inclusion: developing learning and participation in schools. Centre for Studies on Inclusive Education (CSIE).

Carrasco, S., Pàmies, J., Ponferrada, M., Ballestín, B., \& Bertran, M. (2011). Segregación escolar e inmigración en Cataluña: aproximaciones etnográficas. En Garcia Castaño, F. J., \& Carrasco, S. (Eds.), Investigaciones en Inmigración y Educación en España. Homenaje a Eduardo Terrén Lalana (pp. 369-403). Madrid: CIDE-Ministerio de Educación.

Castejón, A. (2017). Expectativas docentes, agrupamiento del alumnado y segregación escolar. Una etnografía en entornos de alta complejidad social en Cataluña. Tesis Doctoral. Departamento de Pedagogía Sistemática y Social, Universitat Autònoma de Barcelona.

Díaz de Rada, Á. (2006). Etnografía y técnicas de investigación antropológica. Madrid: UNED.

Dupriez, V. (2010). Methods of Grouping Learners at School. Paris: UNESCO.

Dupriez, V., \& Dumay, X. (2006). Inequalities in school systems: effect of school structure or of society structure? Comparative Education, 42(2), pp. 243-260. doi: 10.1080/03050060600628074.

Flick, U. (2004). Introducción a la investigación cualitativa. Madrid: Morata.

Gamoran, A. (1992). Synthesis of Research: Is Ability Grouping Equitable? Educational Leadership, 50(2), pp. 11-17.

Gamoran, A. (2009). Tracking and Inequality: New Directions for Research and Practice (No. 2009-6, WCER Working Paper). Madison: University of Wisconsin-Madison, Wisconsin Center for Education Research.

Geertz, C. (1990). La interpretación de las culturas. Barcelona: Gedisa.

González-Motos, S. (2012). Experiencias escolares iniciales del alumnado inmigrantes: experiencias que marcan. Educación XX1, 15(2), pp. 137-158. 
Horn, D. (2009). Age of selection counts: a cross-country analysis of educational institutions. Educational Research and Evaluation, 15(4), pp. 343-366. doi: 10.1080/13803610903087011.

Husén, T. (1973). Implications of IE A Findings for the Philosophy of Comprehensive Education. Harvard University, Cambridge: Paper presented at the Conference on Educational Achievement.

Kelly, S., \& Carbonaro, W. (2012). Curriculum tracking and teacher expectations: evidence from discrepant course taking models. Social Psychology of Education, 15(3), pp. 271-294.

Kutnick, P., Sebba, J., Blatchford, P., Galton, M., \& Thorp, J. (2005). The Effects of Pupil Grouping: Literature Review. University of Brighton, Research Report 688.

Lahire, B. (2008). Un sociólogo en el aula: objetos en juego y modalidades. En Rociles Rubio, M.A., \& Franzé Mudanó, A. (Coords.), ¿Es la escuela el problema? Perspectivas socio-antropológicas de etnografía y educación (pp. 49-60). Madrid: Trotta.

Oakes, J. (1982). The reproduction of inequity: The content of secondary school tracking. The Urban Review, 14(2), pp. 107-120. doi: 10.1007/BF02174647.

Pàmies, J. (2013). El impacto de los agrupamientos escolares. Los espacios de aprendizaje y sociabilidad de los jóvenes de origen marroquí en Barcelona. Revista de Educación, 362 (Septiembre-diciembre 2013), pp. 133-158.

Pàmies, J., Carrasco, S., \& Casalta, V. (2014). Más allá del extrañamiento. Negociaciones y rupturas, o cuando el maestro deviene etnógrafo. En Osuna C., \& Mata-Benito, P. (Eds.), Gestión de acceso al campo y devolución de los resultados: Contextos múltiples de socialización y aprendiraje. Un análisis desde la etnografía de la educación (pp. 19-24). Traficantes de Sueños.

PISA (2015). PISA 2015 Database. Recuperado de: http://www.oecd.org/pisa/.

Ponferrada, M. (2009). Efectos escolares y sociales de la separación por niveles en un instituto de secundaria de la periferia de Barcelona. Papeles de Economía Española, 119, pp. 69-83.

Rubio, M. (2013). Construyendo diferencias desde las retóricas de la igualdad: el caso del alumnado denominado latinoamericano en la etapa de Educación Secundaria Obligatoria. Granada: Universidad de Granada.

Serra, C. (2001). Identitat, racisme i violència. Les relacions interètniques en un institut català. Girona: Universitat de Girona.

Slavin, R.E. (1988). Synthesis of research on grouping in elementary and secondary schools. Educational leadership, 46(1), pp. 67-77.

Sukhnandan, L., \& Lee, B. (1998). Streaming, setting and grouping by ability: a review of the literature. National Foundation for Educational Research.

Wolcott, H.F. (1993). Sobre la intención etnográfica. En Velasco Maillo, H. M., García Castaño, F. J., \& Díaz de Rada Brun, Á. (Coords.), Lecturas de Antropología para Educadores (pp. 127-145). Madrid: Trotta. 